\title{
Declining Presence of Family Physicians in Hospital-Based Care: A Major Concern or Totally Makes Sense?
}

Peter J. Carek, MD, MS

(J Am Board Fam Med 2019;32:768-770.)

Keywords: Continuity of Patient Care, Family Physicians, Health Policy, Hospital Medicine, Inpatients, Leadership, Medical Education, Pay for Performance, Residency

Comprehensiveness along with continuity, coordination, and first contact serve as the 4 pillars of primary care. ${ }^{1}$ Because of their training, family physicians (FPs) are specialists qualified to treat most ailments and provide comprehensive health care to people of all ages. ${ }^{2}$ This comprehensive care to patients regardless of setting is an integral aspect of a highly functioning primary care system.

In this issue, Jetty et $\mathrm{al}^{3}$ provide additional evidence demonstrating the contracting scope, or comprehensiveness, of practice by FPs. Data from the 2013 to 2017 American Board of Family Medicine Certification Examination Registration Questionnaire was used to examine trends in hospital care. Among over 46,000 respondents, the share of FPs reporting hospital care decreased by about $26 \%$, from $34.1 \%$ in 2013 to $25.2 \%$ in 2017 . Basically, this policy brief indicates that the proportion of FPs providing inpatient care has decreased since 2013, leaving approximately 1 in 4 FPs practicing hospital medicine. As noted by the authors and observed in other services often associated with comprehensive care (ie, prenatal care, home visits, nursing home care, and obstetric care), this new information further supports the evidence demonstrating a contracting scope of practice among FPs. ${ }^{4}$

From the Department of Community Health and Family Medicine, College of Medicine, University of Florida, Gainesville, FL.

Funding: none.

Conflict of interest: none declared.

Corresponding author: Peter J. Carek, MD, MS, Department of Community Health and Family Medicine, College of Medicine, University of Florida, PO Box 100237, Gainesville, FL (E-mail: carek@ufl.edu).

\section{What Are Possible Factors?}

In previous studies, no clearly identified cause for a narrowing of scope of practice for FPs has been determined. ${ }^{5}$ The potential forces behind this change are most likely numerous and multifactorial considering the complexity of our health care system and evolving needs of patients, communities, and physicians. Possible factors previously discussed include training and individual preferences as well as external factors such as poor insurance reimbursement, employment contract restrictions, market incentives, or time constraints as a result of increasingly complex patients. ${ }^{6-9}$

\section{Residency Training}

The Accreditation Council for Graduate Medical Education (ACGME) Review Committee for Family Medicine (RC-FM) guidelines have been fairly stable throughout this time period regarding the amount of training in the inpatient setting. Specifically, the ACGME RC-FM guidelines in 2014 and 2019 both included the requirement that residents must have at least the equivalent of 6 months of inpatient care experience and residents must maintain continuity of patient across settings, including caring for continuity patients in the hospital. This requirement was further standardized to include number of patients seen in this setting during the required time period. While the time in the hospital setting has remained the same, other factors of residency training such as the number of patients available for care by an individual resident; the impact of duty hours limiting the total number of hours and increasing the number of transitions of 
care episodes, or the continuity of care provided by a resident to a specific patient; the quality of training in the inpatient setting; or a greater emphasis on the care of patients on an outpatient basis may be influencing residency program graduate's overall experience with inpatient in such a manner as to decrease their ability or willingness to provide hospital care.

\section{Health Systems}

The practice or health system employing FPs may have varying expectations and may be emphasizing ambulatory care to a greater degree. The emergence of hospitalists has either been a factor or a consequence of declining FP presence in the hospital setting. ${ }^{10}$ As noted in a study by Meltzer et $\mathrm{al},{ }^{10}$ "the declining inpatient activity of generalist physicians has not resulted primarily from hospitalists 'crowding out' traditional generalists from inpatient care." On the contrary, their study found that that increases in the overall number of generalists and decline in both hospitalization and length of stay reduced the volume of, and probably the incentives for, generalist inpatient activity over the year just before the rapid growth of hospitalists.

\section{Individual FP}

A majority of FPs (54\%) are using hospitalist services. ${ }^{11}$ While working the same number of hours, many FPs have decided to focus their professional time on providing outpatient care only. ${ }^{4}$ Furthermore, many FPs have discontinued providing direct inpatient care and many feel this does not impact overall quality of care. In a study from Canada, only $47.3 \%$ of FPs surveyed believed the quality of care would suffer (compared with $92.1 \%$ in 1977 and $87.5 \%$ in 1997) if they were not involved in the care of their patients in the hospital. ${ }^{12}$ The reasons for this change in opinion may be related to the previously mentioned decreased volume of hospitalized patients as we as differing communication methods including a broader use of electronic health records.

\section{Community and Population Needs}

While not included in the pillars of primary care, FPs are obligated to care for individual patients as well as for the larger community they service. To do so and with the limits of resources and time, an FP must balance these competing priorities in such a manner as to work both efficiently and effectively. As noted in the ecology of health care, a substantial majority of health care is provided on an outpatient basis. ${ }^{13}$ Furthermore, recent data from the Center for Disease Control and Prevention (CDC) indicates that the number of outpatient visits to physicians $(883,725,000$ physician office visits in 2016) is significantly larger than the number of hospital admissions (approximately 35,061,000 in 2015). ${ }^{14,15}$ Due to the scale of these differing numbers and a host of other factors (ie, cost, efficiency, and convenience to name a few), the community may be requiring the FP to spend more time addressing ambulatory medicine and, as a consequence, less focus on inpatient care. As such, the trend in FPs proving outpatient care only is logical and reasoned.

\section{What Really Is Comprehensiveness?}

Starfield ${ }^{1}$ stated that "comprehensiveness means that all problems in the population should be cared for in primary care (with short-term referral as needed), except those that are too unusual (generally a frequency of less than 1 or 2 per thousand in the population served) for the primary care practitioner or team to treat competently." Conversely, Starfield also noted that "the benefits of the other 2 main attributes of good primary care (comprehensiveness and coordination) are less well documented." ${ }^{1}$ In addition, how the problems are "cared for" in primary care is not specifically stated. Just as some medical problems are being delegated to or require the assistance of specialists, many FPs are now delegating or seeking the assistance of other physicians and health care provides to care for their patients in the hospital setting.

A delineation of the specific activities or services provided by a family or other primary care physician that provides the comprehensiveness required for optimal patient and population health and wellness has not been developed, studied, and implemented. Historically, the generalist physician appeared to provide a much broader scope of practice in terms of services before the era of specialization than the current scope for FPs. This assumption is tethered by the relatively limited number of overall physician services that could be offered as diagnostic and treatment options were not as vast as they are today. In this historic context, scope of practice changes, should not be considered stagnant, and probably require constant evaluation and assessment.

Scope of practice also differs among countries and geographic regions. Generalist physicians in the 
United Kingdom (UK) are less likely to refer patients to a specialist than their US counterparts. While this finding is influenced by the low availability of specialists in the UK, this finding suggests that the UK physicians have a broader scope of practice at least in the ambulatory care setting. ${ }^{16,17}$ In a study of Canadian FPs and General Practitioners, geographic factors of provincial division and whether or not the population served was rural explained $30.5 \%$ of the variation in the scope of practice. ${ }^{18}$

A change in the scope of practice does not always mean narrowing. Using the provision of inpatient care as a marker of comprehensiveness may be not be sufficient or even necessary as a majority of care needed by our patient population occurs in the ambulatory care setting. ${ }^{13}$ In addition, services and activities not currently considered in the scope of practice of an FP may need to be reconsidered. Moving forward, the scope of practice for FPs will change due to differing payment methods, innovative technology, changing individual patient and community needs, increasing emphasis on addressing issues of wellness, and other factors. For instance, improved communication technology and enhanced payment systems may allow and encourage better interaction between an FP, patient and inpatient care physicians without the FP having provide direct care or present in-person to the hospital.

While the authors encourage better research on the impact that practice settings may have on patients and health systems, additional research is needed to determine what are the factors or services attributed to comprehensiveness that improve the quality of care provided by FPs. Comprehensive shares the same Latin origin as comprehend. As FPs, we need to better understand the services needed by our patients and communities that provide the comprehensive care and appropriate services that leads to better health outcomes.

\section{References}

1. Starfield B, Shi L, Macinko J. Contribution of primary care to health systems and health. Milbank Q 2005;83:457-502.

2. American Academy of Family Physicians. Family medicine specialty. Available from: https://www.aafp. org/about/the-aafp/family-medicine-specialty.html. Accessed September 9, 2019.

3. Jetty A, Jabbarpour Y, Petterson S, Eden A, Ba- zemore A. The declining presence of family physicians in hospital-based care. J Am Board Fam Med 2019;32(6):771-772.

4. Carek PJ, Diaz V, Dickerson LM, Peterson L, Johnson S. Preparation for practice in family medicine: before and after duty hours. Fam Med 2012;44:539-544.

5. Coutinho AJ, Cochrane A, Stelter K, Phillips RL Jr, Peterson LE. Comparison of intended scope of practice for family medicine residents with reported scope of practice among practicing family physicians. JAMA 2015;314:2364-2372.

6. Tong ST, Makaroff LA, Xierali IM, et al. Proportion of family physicians providing maternity care continues to decline. J Am Board Fam Med 2012;25:270-271.

7. Bazemore AW, Makaroff LA, Puffer JC, et al. Declining numbers of family physicians are caring for children. J Am Board Fam Med 2012;25:139-140.

8. Xierali IM, Puffer JC, Tong ST, Bazemore AW, Green LA. The percentage of family physicians attending to women's gender-specific health needs is declining. J Am Board Fam Med 2012;25:406-407.

9. Carek PJ. Potentially alarming trends in the scope of practice for family physicians. J Am Board Fam Med 2018;31:178-180.

10. Metzer DO, Chung JW. U.S. trends in hospitalization and generalist physician workforce and the emergence of hospitalists. J Gen Intern Med 25:453-459.

11. Ivins D, Blackburn B, Peterson LE, et al. A majority of family physicians use a hospitalist service when their patients require inpatient care. J Prim Care Community Health 2015;6:70-76.

12. Neimanis I, Woods A, Zizzo A, et al. Role of family physicians in an urban hospital: tracking changes between 1977, 1997, and 2014. Can Fam Physician 2017; 63:221-227.

13. Green LA, Fryer GE Jr, Yawn BP, Lanier D, Dovey SM. The ecology of medical care revisited. N Engl J Med 2001;344:2021-2025.

14. Center for Disease Control and Prevention. Ambulatory health care data. Available from: https://www.cdc.gov/ nchs/ahcd/index.htm. Accessed September 10, 2019.

15. Center for Disease Control and Prevention. Hospital admission, average length of stay, outpatient visits, and outpatient surgery, by type of ownership and size of hospital: United States, selected years 19752015. Available from: https://www.cdc.gov/nchs/ data/hus/2017/082.pdf. Accessed September 10, 2019.

16. Grumbach K. To be or not to be comprehensive. Ann Fam Med 2015;13:204-205.

17. Forrest CB, Majeed A, Weiner JP, Carroll K, Bindman AB. Comparison of specialty referral rates in the United Kingdom and the United States: retrospective cohort analysis. BMJ 2002;325:370-371.

18. Wong E. Predicting the scope of practice of family physicians. Can Fam Physician 2010;56(6):e219e225. 